\title{
Oral Manifestations of Nutritional Deficiencies: Single Centre Analysis
}

\author{
Vladimíra Radochová1,*, Radovan Slezák¹, Jakub Radocha²
}

\begin{abstract}
Introduction: Oral manifestations of deficiency of iron, vitamin B12 and folic acid are thought to be common. Prevalence of these deficiencies among patients with compatible symptoms is not well known. The goal of this study was to summarize evidence from a dental practice of iron, vitamin B12 and folic acid deficiency in patients presenting with compatible oral manifestations.

Methods: 250 patients who presented with burning mouth syndrome, angular cheilitis, recurrent aphthous stomatitis, papillar atrophy of the tongue dorsum or mucosal erythema were identified. Patients underwent clinical examination, and the blood samples were taken. Results: 250 patients ( 208 females; 42 males, mean age 44.1 years) with at least one corresponding symptom or sign were identified. The nutritional deficiency of one or more nutrients was found in 119 patients (47.6\%). Seven times more females than males were noted to have one type of deficiency (104 females, 15 males). Iron deficiency as defined was diagnosed in 62 patients (24.8\%), vitamin B12 or folic acid deficiency in 44 patients (17.6\%) and both deficiencies (iron + vitamin B12/folic acid) in 13 patients (5.2\%). The only predictive factor was gender and only for iron deficiency. The presence of more than one deficiency was noted in 10 patients (4.9\%).

Conclusion: The most commonly observed deficiency in dental practice over the course of 11 years was an iron deficiency in the female population. Age, diet and reported co-morbidities did not show statistically significant predictable value in recognizing these deficiencies.

\section{KEYWORDS}

anemia; oral manifestations; iron deficiency; vitamin B12; folic acid

\section{AUTHOR AFFILIATIONS}

${ }^{1}$ Department of Dentistry, Faculty of Medicine in Hradec Králové, Charles University, and University Hospital Hradec Králové, Sokolská 581, 50005 Hradec Králové, Czech Republic

2 4th Department of Internal Medicine-Hematology, Faculty of Medicine in Hradec Králové, Charles University, and University Hospital Hradec Králové, Sokolská 581, 50005 Hradec Králové, Czech Republic

* Corresponding author: Department of Dentistry, University Hospital, Sokolská 581, 50005 Hradec Králové, Czech Republic, e-mail: vladimira.radochova@fnhk.cz
\end{abstract}

Received: 1 April 2020

Accepted: 5 June 2020

Published online: 1 October 2020

Acta Medica (Hradec Králové) 2020; 63(3): 95-100

https://doi.org/10.14712/18059694.2020.25

(c) 2020 The Authors. This is an open-access article distributed under the terms of the Creative Commons Attribution License (http://creativecommons.org/licenses/by/4.0), which permits unrestricted use, distribution, and reproduction in any medium, provided the original author and source are credited. 


\section{INTRODUCTION}

Vitamin and mineral nutritional deficiencies represent some of the most frequent disorders worldwide (1). Dental practitioners are often some of the first healthcare providers to observe oral manifestations of burning mouth, mucosal erythema or papillary atrophy of the tongue dorsum. The iron, folic acid and vitamin B12 deficiencies represent common challenging scenarios in routine dental practice. According to the World Health Organization (WHO), iron deficiency represents a common medical challenge with about $80 \%$ of anemias represented by iron deficiency, and $30 \%$ of the world population is at risk of developing iron deficiency (2). A vegetarian or vegan diet might influence the prevalence of nutrient deficiencies (3).

The clinical signs observed, and symptoms associated with oral cavity are often non-specific and may mimic or overlap with other types of deficiencies or disorders. The most frequently reported oral cavity symptoms of iron, vitamin B12 and folic acid deficiency are pale mucous membranes, erythema, glossitis, recurrent aphthous stomatitis, angular cheilitis and oral candidiasis $(4,5)$. Angular cheilitis and glossitis are mentioned as some of the common symptoms of iron deficiency (6). However, these two clinical signs may represent other pathology. The red beef tongue and burning mouth are more often reported to be related to vitamin B12 deficiency (7). The burning mouth, burning tongue, paresthesia and dysesthesia of the oral cavity often represent non-specific symptoms with nutritional deficiencies identified as some of the more common systemic factors (8).

The diagnosis of a nutritional or vitamin deficiency is based on patients' history, physical examination and laboratory findings. When oral signs and symptoms are identified, iron, vitamin B12 and folic acid deficiencies are commonly considered in differential diagnoses. The complete blood count, iron storage estimation and levels of vitamin B12 and folic acid represent necessary armamentarium for examination and development of differential diagnoses. These tests and exams are not commonly conducted by a general dentist. Furthermore, in large metropolitan or medical centre areas, the patient may easily be referred to an oral medicine specialist. This is not always possible in more rural areas or where there is limited access to an oral medicine specialist.

The goal of this retrospective study was to summarize clinical-based evidence from a dental practice of iron, vitamin B12 and folic acid deficiency in patients presenting with oral manifestations as an effort to improve health care delivery and provide information to help decision making and management of care with future patients.

\section{PATIENTS AND METHODS}

The patients who were referred to the consultation to the Department of Dentistry (tertiary dental clinic) University Hospital Hradec Kralove from January 2007 to December 2018 with one or more clinical symptoms or clinical signs were identified. The inclusion criteria for patients are summarized in Table 1 . Patients with a known diagnosis of anemia were excluded from the study.
Tab. 1 Significant clinical signs and symptoms.

\begin{tabular}{|l|l|}
\hline Objective signs & $\begin{array}{l}\text { Subjective symptoms } \\
\text { and history }\end{array}$ \\
\hline $\begin{array}{l}\text { glossitis with or without } \\
\text { atrophic papillae }\end{array}$ & burning mouth syndrome \\
\hline $\begin{array}{l}\text { generalized or localized mu- } \\
\text { cosal erythema }\end{array}$ & history of angular cheilitis \\
\hline angular cheilitis & $\begin{array}{l}\text { history of recurrent aphthous } \\
\text { stomatitis }\end{array}$ \\
\hline aphthous stomatitis & history of recurrent candidosis \\
\hline oral candidosis & \\
\hline
\end{tabular}

A retrospective chart review was conducted of the identified patients. The research protocol was approved by the institutional review board and ethical committee.

The parameters of interest included: patient age, sex, special diets, the initial date of appointment, medical, dental, social, and family history; review of medications, allergies and medical co-morbidities. Initial intraoral and extraoral examination findings at the time of patient presentation, patient-reported symptoms and signs (Table 2), complete blood count with differential leukocyte count, vitamin B12 level, folic acid level, ferritin, serum iron and total iron-binding capacity, basic chemistry panel especially liver and kidney function tests, minerals, glucose) conducted as a routine practice to exclude potential frequent systemic disease.

Tab. 2 Frequency of specific deficiencies.

\begin{tabular}{|l|l|l|}
\hline & N & (\%) \\
\hline $\begin{array}{l}\text { Total No. Of patients with symptoms } \\
\text { (in Table 1) }\end{array}$ & 250 & \\
\hline Females & 208 & 83.2 \\
\hline Males & 42 & 16.8 \\
\hline Mean age (years) & & \\
\hline Females & 46.4 & \\
\hline Males & 51.7 & \\
\hline History of systemic disease & & \\
\hline Hypothyroidism & 20 & 8.0 \\
\hline Autoimmune disease $*$ & 11 & 4.4 \\
\hline Arterial hypertension & 7 & 2.8 \\
\hline Cancer (breast) & 3 & 1.2 \\
\hline Diabetes mellitus & 3 & 1.2 \\
\hline Other & 4 & 1.6 \\
\hline Nutrient deficient patients & 131 & 5.2 \\
\hline Total number of deficiencies & 119 & 47.6 \\
\hline Iron deficiency & 62 & 24.8 \\
\hline Vitamin B12 and/or folic acid deficiency & 44 & 17.6 \\
\hline Iron deficiency+ B12 and/or folic acid & 13 & 5.2 \\
\hline Total number without deficiency & 131 \\
\hline Fis & & \\
\hline & & \\
\hline
\end{tabular}

* Includes rheumatoid arthritis, Sjögren syndrome, bronchial asthma. 
The presence of iron deficiency was defined as serum ferritin level of less than $20 \mu \mathrm{g} / \mathrm{l}$. Vitamin B12 deficiency was defined as serum levels less than $150 \mathrm{pmol} / \mathrm{l}$ and less than $9 \mathrm{nmol} / \mathrm{l}$ for folic acid deficiency. The latent deficiency was defined as presence of deficiency without anemia or other changes in the blood count. It was taken in account in the same manner as deficiency with anemia.

The continuous parameters of interest were summarized with means, medians, ranges. The categorical parameters were summarized with percentages and frequencies. Chi-square and Fisher's exact test was used to evaluate comparisons among parameters of interest. Data were analyzed using Microsoft Excel 2007 (Microsoft, USA) and MedCalc 9.5.2.0 (MedCalc, Belgium). P-values <.05 were considered statistically significant, and all tests were 2-sided.

\section{RESULTS}

\section{PATIENT DEMOGRAPHICS}

A total of 250 patients (208 females; 42 males, mean age 44.1 years) were identified. No patient was vegetarian or vegan. The nutritional deficiency of one or more nutrients was found in 119 patients (47.6\%). Seven times more females than males were noted to have one type of deficiency (104 females, 15 males). Iron deficiency as defined was diagnosed in 62 patients $(24.8 \%)$, vitamin B12 or folic acid deficiency in 44 patients (17.6\%) and both deficiencies (iron + vitamin B12 and/or folic acid) in 13 patients (5.2\%). Patients' characteristics are given in detail in Table 2. Females were more likely affected by iron deficiency than males $(p=0.020)$.

\section{CLINICAL SIGNS AND SYMPTOMS}

Distribution of each symptom and sign is shown in Table 3 , and in specific groups of patients with corresponding deficiency is shown in Table 4. Comparison of a group of patients with and without deficiency related to symptom frequency is shown in Table 5. Erythema of the mucosa was present more frequently in patients with a deficiency compared to those without $(29.4 \%$ versus $10.7 \%, \mathrm{p}<0.001)$. Angular cheilitis was significantly more associated with the presence of deficiency (36.1\% with and $17.6 \%$ without deficiency, $p=0.001)$. The common clinical sign noted in patients with an identified deficiency was papillary atrophy regardless of deficiency type (31.9\% with and $9.9 \%$ without deficiency, $p<0.001$ ). Presence of burning mouth and recurrent aphthous stomatitis was not significantly different among patients with and without deficiency, see Table 6 for details.

Tab. 3 Frequency of symptoms and signs.

\begin{tabular}{|l|l|l|}
\hline Symptom present & N & (\%) \\
\hline Burning mouth syndrome & 129 & 51.6 \\
\hline Recurrent aphthous stomatitis & 89 & 35.6 \\
\hline Angular cheilitis & 66 & 26.4 \\
\hline Papillar atrophy of tongue dorsum & 51 & 20.4 \\
\hline Erythema & 49 & 19.6 \\
\hline Candidosis & 9 & 3.6 \\
\hline
\end{tabular}

Tab. 5 Differences in symptoms in patients with and without deficiency.

\begin{tabular}{|c|c|c|c|}
\hline & $\mathbf{N}$ & $\%$ & $p$ value \\
\hline \multicolumn{4}{|l|}{ Erythema } \\
\hline Deficiency & $35 / 119$ & 29.4 & $<0.001$ \\
\hline No deficiency & $14 / 131$ & 10.7 & \\
\hline \multicolumn{4}{|l|}{ Burning } \\
\hline Deficiency & $62 / 119$ & 52.1 & 0.981 \\
\hline No deficiency & 67/131 & 56.3 & \\
\hline \multicolumn{4}{|l|}{ Aphthae } \\
\hline Deficiency & $38 / 119$ & 31.9 & 0.307 \\
\hline No deficiency & $51 / 131$ & 38.9 & \\
\hline \multicolumn{4}{|l|}{ Papillar atrophy } \\
\hline Deficiency & $38 / 119$ & 31.9 & $<0.001$ \\
\hline No deficiency & 13/131 & 9.9 & \\
\hline \multicolumn{4}{|c|}{ Angular cheilitis } \\
\hline Deficiency & $43 / 119$ & 36.1 & 0.001 \\
\hline No deficiency & 23/131 & 17.6 & \\
\hline
\end{tabular}

Tab. 4 Symptoms according to deficiency.

\begin{tabular}{|l|l|l|l|l|l|l|l|l|l|l|l|l|}
\hline & Burning & $\%$ & $\begin{array}{l}\text { Recurrent } \\
\text { aphthae }\end{array}$ & $\%$ & $\begin{array}{l}\text { Angular } \\
\text { cheilitis }\end{array}$ & $\%$ & Erythema & $\%$ & $\begin{array}{l}\text { Papillar } \\
\text { atrophy }\end{array}$ & $\%$ & Candidosis & $\%$ \\
\hline Overall (N) & 129 & & 89 & & 66 & & 49 & & 51 & & 9 \\
\hline Males & 18 & 14.0 & 18 & 20.2 & 6 & 9.1 & 5 & 10.2 & 4 & 7.8 & 2 \\
\hline Females & 111 & 86.0 & 71 & 79.8 & 60 & 90.9 & 44 & 89.8 & 47 & 92.2 & 7 \\
\hline Iron deficiency & 34 & 26.4 & 25 & 28.1 & 29 & 43.9 & 19 & 38.8 & 28 & 54.9 & 3 \\
\hline B12 deficiency & 34 & 26.4 & 14 & 15.7 & 18 & 27.3 & 21 & 42.9 & 16 & 31.4 & 4 \\
\hline Folic acid deficiency & 6 & 4.7 & 5 & 5.6 & 3 & 4.5 & 5 & 10.2 & 3 & 53.3 \\
\hline None & 67 & 51.9 & 51 & 57.3 & 23 & 34.8 & 14 & 28.6 & 13 & 25.9 & 2 \\
\hline
\end{tabular}


Tab. 6 Comparison of symptoms in iron and vitamin B12/folic acid deficiency.

\begin{tabular}{|l|l|l|l|}
\hline \multicolumn{2}{|l|}{ N } & $\%$ & p value \\
\hline Burning & $34 / 62$ & 54.8 & \\
\hline Iron deficiency & 0.0002 \\
\hline B12/folic acid deficiency & $40 / 44$ & 90.9 & \\
\hline Papillar atrophy of the tongue dorsum & 0.997 \\
\hline Iron deficiency & $28 / 62$ & 45.2 & \\
\hline B12/folic acid deficiency & $19 / 44$ & 43.2 & \\
\hline Angular cheilitis & & & 0.92 \\
\hline Iron deficiency & $29 / 62$ & 46.8 & \\
\hline B12/folic acid deficiency & $21 / 44$ & 47.7 & \\
\hline Aphthae & & 0.924 \\
\hline Iron deficiency & $25 / 62$ & 40.3 & \\
\hline B12/folic acid deficiency & $19 / 44$ & 43.2 & \\
\hline Erythema & & & 0.007 \\
\hline Iron deficiency & $19 / 62$ & 30.6 & \\
\hline B12/folic acid deficiency & $26 / 44$ & 59.1 & \\
\hline
\end{tabular}

\section{IRON, VITAMIN B12, FOLIC ACID}

The most common deficiency was iron deficiency representing $52.1 \%$ of all pathologies followed by vitamin B12 and/or folic acid deficiency (36.9\%). Both deficiencies (iron + vitamin B12 and/or folic acid) represented 10.9\% of all deficiencies. Anemia in the blood count was found in more than half of patients with iron, vitamin B12 or folic acid deficiency $(n=61 / 119,51.3 \%)$.

\section{CLINICAL MANAGEMENT AND FOLLOW UP}

Local treatment was advised for patients with candidosis (antifungal oral rinses) and topical treatment with hydrocortisone, natamycin and neomycin ointment was advised for patients with angular cheilitis. The patients were referred to the general practitioner when the deficiency was found. Supplementation of missing nutrient, search for the underlying cause was recommended. Patient counselling of such deficiencies was completed together with a discussion of their impact on overall health. None of the patients whose symptoms were due to nutrient deficiency was in need of additional referrals for further follow up to our department. Results of supplementation by the general practitioner are not available.

\section{DISCUSSION}

Nutritional deficiencies represent a significant medical challenge even in the contemporary European population. Diet habits and socioeconomic status may contribute to the development of such problems (9). Women are generally at higher risk of iron deficiency because of their regular iron loss during the menstrual cycle and pregnancy (overall, 58 females with iron deficiency compared to only four males in the current study). One of the most significant studies by Viñas published in 2011 described the prevalence of micronutrient deficiencies in the European population. $21-30 \%$ of adult males from Finland and adult females from Ireland (both countries with high social and economic standards) had insufficient vitamin B12 intake. The prevalence of vitamin B12 deficiency increases with age as well. It reached $19 \%$ in a Swiss population of seniors above 80 years of age (10). Another study from Ireland described only $2.7 \%$ of vitamin B12 deficiency in the adult population (11). Results of our group of folic acid and vitamin B12 did not vary in age compared to the group without deficiency. Older patients might especially suffer from folic acid deficiency (12). Iron deficiency seems to affect a more extensive age range. Children as young as 12 to 36 months of age could have up to $11.8 \%$ of iron deficiency (13). Adolescent children between 12 and 17 years of age have iron deficiency present in $17.6 \%$ across Europe (14). Finally, the prevalence of iron deficiency anemia can reach up to $19.9 \%$ in the adult European population (15). The age of our iron-deficient patients (mean age 41.9 years) was significantly lower than in those without deficiency.

Since the prevalence of nutritional deficiencies is high, their presence is expected to be high as well in the general population. The first signs and symptoms of these deficiencies do not necessarily need to be anemia and often manifest themselves in other organ symptoms. Graells published a small pivotal study of 4 patients with Hunter glossitis and normal blood count. The organ symptoms in all 4 patients preceded the development of anemia (16). In the presented study, $48.7 \%$ of patients $(N=58)$ had a deficiency without any change in the blood count. These findings are consistent with the study of Asian population where a total of 149 patients with vitamin B12 and iron deficiency revealed normal blood counts in $36.2 \%$ of patients matching almost entirely results from our Czech sample (17).

Burning mouth is one of the most frequent signs that bring patients to dental medicine clinics. It represents a wide variety of diseases ranging from benign to severe disorders. The exact etiology of burning mouth syndrome cannot be frequently identified (18). Nutrition deficiencies and anemia represent the frequent and often reversible cause of burning mouth. Lin et al. described a decrease of hemoglobin in a cohort of 399 Asian patients with the burning mouth in $22.3 \% .20 .3 \%$ of these 399 patients had iron deficiency, $2.5 \%$ vitamin B12 deficiency and $1.5 \%$ folic acid deficiency (19). Very similar findings are shown by the current study, where $24.8 \%$ of patients had an iron deficiency in a slightly smaller European population.

Similarly, our current data showed a more significant portion of patients with vitamin B12 and the folic acid deficiency (17.6\%). An Italian study showed $11 \%$ of vitamin B12 and $12.5 \%$ of folic acid deficiency in patients with burning mouth syndrome (20). There are apparent geographic differences in the prevalence of each deficiency. Even subtle changes in mean cell erythrocyte volume can be a sign of deficiency. All patients $(100 \%)$ with increases mean cell volume had burning mouth in the study by 
Chang published in 2015. 66.7\% of these patients had concomitants atrophic glossitis (21).

The oral symptoms are not similar for various deficiencies. While the most frequent symptom (burning mouth) is similarly distributed among patients with and without underlying deficiency, objective signs differ in groups of patients with and without deficiency in our cohort of patients. Likewise, the most frequent sign described is angular cheilitis (58\%) and papillar atrophy of the tongue dorsum (42\%) in a smaller cohort of patients with oral symptoms from 2004 as reported by Lu et al. (4). The patients with iron deficiency represented $40 \%$ of symptomatic patients and vitamin B12 20\% (4). Analysis of our group revealed one fourth (24\%) of patients with iron deficiency and $11.7 \%$ of patients with vitamin B12 deficiency. Very similar findings were observed in a study by Wu et al. (22) The authors described the cohort of iron-deficient patients showing papillar atrophy present in $26.7 \%$ of patients with iron deficiency compared to $54.9 \%$ in our group.

According to Sun et al., patients with papillar atrophy more frequently have iron deficiency (26.7\%) than vitamin B12 deficiency (7.4\%) (23). Andrès et al. described atrophic glossitis in $62 \%$ of hospitalized patients who were found to have vitamin B12 deficiency which is more compared to our study (43.2\%) (24). Recurrent aphthous stomatitis is also a frequent finding. Iron deficiency can be identified in around $20 \%$ of patients with aphthous stomatitis which is a bit lower than $28.1 \%$ in our group (25). Kozlaka et al. found that patients with recurrent aphthous stomatitis have a lower daily intake of vitamin B12 compared to healthy population (26). We observed a very low prevalence of oral candidiasis in our group. Recently published data by Lu showed the prevalence of oral pseudomembranous and erythematous candidiasis around $40 \%$ in patients in a similar cohort of patients (27). The atrophic glossitis can have the very similar clinical picture, and the deficiency can be overlooked (28).

Reversal of symptoms by supplementation of missing nutrients has been shown to be effective in a cohort of 399 patients (18). The burning mouth disappeared within 5-10 months after supplementation of missing nutrient in virtually all affected patients (29). None of our patients with revealed deficiency came back with the same symptoms after the recommended treatment.

\section{CONCLUSION}

Nutritional deficiencies and their symptoms are very frequent and commonly present without corresponding anemia. The most common deficiency identified in our cohort was iron deficiency with an expected female predominance. Other deficiencies were evenly distributed among males and females. The diagnosis of the deficiency might also lead to the identification of potentially life-threatening underlying diseases and maybe the first guide to the correct diagnosis of systemic disease and treatment. The dentists should be familiar with the above to identify the signs and symptoms, forcing the proper evaluation adequately.

\section{ACKNOWLEDGEMENTS}

The work was supported by the project PROGRES Q40/08 and Q40/13 (Charles University, Faculty of Medicine in Hradec Králové) and by the Czech Health Research Council, Ministry of Health of the Czech Republic and $\mathrm{MH}$ CZ - DRO (UHHK, 00179906).

\section{CONFLICTS OF INTEREST}

None declared.

REFERENCES:

1. GlobalHealthRisks_report_full.pdf [Internet]. Available at: http:// www.who.int/healthinfo/global_burden_disease/ GlobalHealthRisks _report_full.pdf

2. WHO | Worldwide prevalence of anaemia 1993-2005 [Internet]. WHO. Available at: http://www.who.int/nutrition/publications /micronutrients/anaemia_iron_deficiency/9789241596657/en/

3. Barr SI. Vegetarian diets. World Rev Nutr Diet 2015; 111: 53-7.

4. Lu S-Y, Wu H-C. Initial diagnosis of anemia from sore mouth and improved classification of anemias by MCV and RDW in 30 patients. Oral Surg Oral Med Oral Pathol Oral Radiol Endod 2004; 98: 679-85.

5. Adeyemo TA, Adeyemo WL, Adediran A, Akinbami AJA, Akanmu AS. Orofacial manifestations of hematological disorders: anemia and hemostatic disorders. Indian J Dent Res 2011; 22: 454-61.

6. Long RG, Hlousek L, Doyle JL. Oral manifestations of systemic diseases. Mt Sinai J Med N Y 1998; 65: 309-15.

7. Greenberg MS. Clinical and histologic changes of the oral mucosa in pernicious anemia. Oral Surg Oral Med Oral Pathol 1981; 52: 38-42.

8. Coculescu EC, Tovaru S, Coculescu BI. Epidemiological and etiological aspects of burning mouth syndrome. J Med Life 2014; 7: 305-9.

9. Huffman FG, Vaccaro JA, Zarini GG, Biller D, Dixon Z. Inadequacy of micronutrients, fat, and fiber consumption in the diets of Haitian-, African- and Cuban-Americans with and without type 2 diabetes. Int J Vitam Nutr Res 2012; 82: 275-87.

10. Risch M, Meier DW, Sakem B, et al. Vitamin B12 and folate levels in healthy Swiss senior citizens: a prospective study evaluating reference intervals and decision limits. BMC Geriatr 2015; 15: 82.

11. McNamee T, Hyland T, Harrington J, et al. Haematinic deficiency and macrocytosis in middle-aged and older adults. PloS One 2013; 8(11): e77743.

12. Roman Viñas B, Ribas Barba L, Ngo J, et al. Projected prevalence of inadequate nutrient intakes in Europe. Ann Nutr Metab 2011; 59: 84-95.

13. Akkermans MD, van der Horst-Graat JM, Eussen SRBM, van Goudoever JB, Brus F. Iron and Vitamin D Deficiency in Healthy Young Children in Western Europe Despite Current Nutritional Recommendations. J Pediatr Gastroenterol Nutr 2016; 62: 635-42.

14. Ferrari M, Mistura L, Patterson E, et al. Evaluation of iron status in European adolescents through biochemical iron indicators: the HELENA Study. Eur J Clin Nutr 2011; 65: 340-9.

15. Fonseca C, Marques F, Robalo Nunes A, Belo A, Brilhante D, Cortez J. Prevalence of anaemia and iron deficiency in Portugal: the EMPIRE study. Intern Med J 2016; 46: 470-8.

16. Graells J, Ojeda RM, Muniesa C, Gonzalez J, Saavedra J. Glossitis with linear lesions: An early sign of vitamin B12 deficiency. J Am Acad Dermatol 2009; 60: 498-500.

17. Chang JY-F, Wang Y-P, Wu Y-C, Cheng S-J, Chen H-M, Sun A. Blood profile of oral mucosal disease patients with both vitamin B12 and iron deficiencies. J Formos Med Assoc 2015; 114: 532-8.

18. Sun A, Wu K-M, Wang Y-P, Lin H-P, Chen H-M, Chiang C-P. Burning mouth syndrome: a review and update. J Oral Pathol Med 2013; 42: 649-55.

19. Lin H-P, Wang Y-P, Chen H-M, Kuo Y-S, Lang M-J, Sun A. Significant association of hematinic deficiencies and high blood homocysteine levels with burning mouth syndrome. J Formos Med Assoc 2013; 112: 319-25.

20. Sardella A, Lodi G, Demarosi F, Uglietti D, Carrassi A. Causative or precipitating aspects of burning mouth syndrome: a case-control study. J Oral Pathol Med 2006; 35: 466-71.

21. Chang JY-F, Wang Y-P, Wu Y-C, Cheng S-J, Chen H-M, Sun A. Hematinic deficiencies and pernicious anemia in oral mucosal dis- 
ease patients with macrocytosis. J Formos Med Assoc 2015; 114: 736-41.

22. Wu Y-C, Wang Y-P, Chang JY-F, Cheng S-J, Chen H-M, Sun A. Oral manifestations and blood profile in patients with iron deficiency anemia. J Formos Med Assoc 2014; 113: 83-7.

23. Sun A, Lin H-P, Wang Y-P, Chiang C-P. Significant association of deficiency of hemoglobin, iron and vitamin B12, high homocysteine level, and gastric parietal cell antibody positivity with atrophic glossitis. J Oral Pathol Med 2012; 41: 500-4.

24. Andrès $E$, Nachit $M$, Guillet-Thibault J. Oral manifestations of vitamin B12 and B9 deficiencies: a prospective study. J Oral Pathol Med 2016; 45: 154-154.

25. Sun A, Chen H-M, Cheng S-J, et al. Significant association of deficiencies of hemoglobin, iron, vitamin B12, and folic acid and high homocysteine level with recurrent aphthous stomatitis. J Oral Pathol Med 2015; 44: 300-5.

26. Kozlak ST, Walsh SJ, Lalla RV. Reduced dietary intake of vitamin B12 and folate in patients with recurrent aphthous stomatitis. J Oral Pathol Med 2010; 39: 420-3.

27. Lu S-Y. Perception of iron deficiency from oral mucosa alterations that show a high prevalence of Candida infection. J Formos Med Assoc 2016; 115: 619-27.

28. Terai H, Shimahara M. Atrophic tongue associated with Candida. J Oral Pathol Med 2005; 34: 397-400.

29. Sun A, Lin H-P, Wang Y-P, Chen H-M, Cheng S-J, Chiang C-P. Significant reduction of serum homocysteine level and oral symptoms after different vitamin-supplement treatments in patients with burning mouth syndrome. J Oral Pathol Med 2013; 42: 4. 